\title{
Metabolic changes associated with papillary thyroid carcinoma: A nuclear magnetic resonance-based metabolomics study
}

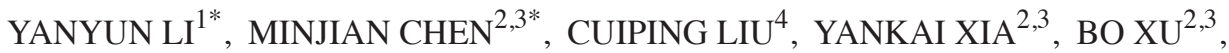 \\ YANHUI HU ${ }^{3}$, TING CHEN ${ }^{5}$, MEIPING SHEN ${ }^{4}$ and WEI TANG ${ }^{1,6}$ \\ ${ }^{1}$ Department of Endocrinology, Jiangyin People's Hospital, School of Medicine, Southeast University, Jiangyin, \\ Jiangsu 214400; ${ }^{2}$ State Key Laboratory of Reproductive Medicine, Institute of Toxicology, School of \\ Public Health, Nanjing Medical University; ${ }^{3}$ Key Laboratory of Modern Toxicology of Ministry of Education, \\ School of Public Health, Nanjing Medical University, Nanjing, Jiangsu 211166; ${ }^{4}$ Department of General Surgery, \\ The First Affiliated Hospital of Nanjing Medical University, Nanjing, Jiangsu 210036; ${ }^{5}$ Department of Science \\ and Education Section, Maternity and Child Care Hospital of Nanjing, Nanjing, Jiangsu 210004; \\ ${ }^{6}$ Department of Endocrinology, Jiangsu Province Official Hospital, Nanjing, Jiangsu 210024, P.R. China
}

Received October 23, 2016; Accepted January 29, 2018

DOI: 10.3892/ijmm.2018.3494

\begin{abstract}
Papillary thyroid carcinoma (PTC) is the most common thyroid cancer. Nuclear magnetic resonance (NMR)-based metabolomic technique is the gold standard in metabolite structural elucidation, and can provide different coverage of information compared with other metabolomic techniques. Here, we firstly conducted NMR based metabolomics study regarding detailed metabolic changes especially metabolic pathway changes related to PTC pathogenesis. ${ }^{1} \mathrm{H}$ NMR-based metabolomic technique was adopted in conjunction with multivariate analysis to analyze matched tumor and normal thyroid
\end{abstract}

Correspondence to: Professor Wei Tang, Department of Endocrinology, Jiangyin People's Hospital, School of Medicine, Southeast University, 163 Shoushan Road, Jiangyin, Jiangsu 214400, P.R. China E-mail: drtangwei@aliyun.com

Professor Meiping Shen, Department of General Surgery, The First Affiliated Hospital of Nanjing Medical University, Nanjing, Jiangsu 210036, P.R. China

E-mail: nj_shenmp@sina.com

*Contributed equally

Abbreviations: PTC, papillary thyroid carcinoma; NMR, nuclear magnetic resonance; KEGG, Kyoto Encyclopedia of Genes and Genomes; HMDB, human metabolome database; PCA, principal component analysis; PLS-DA, partial least squares discriminant analysis; OPLS-DA, orthogonal partial least-squares discriminant analysis; SMPDB, Small Molecule Pathway Database; VLDL, verylow-density lipoprotein; LDL, low-density lipoprotein; MS, mass spectrometry; GC-MS, gas chromatography-mass spectrometry; VIP, variable importance in the projection; FAs, follicular adenomas; CPMG, Carr-Purcell-Meiboom-Gill

Key words: papillary thyroid carcinoma, metabolomics, nuclear magnetic resonance, pathogenesis tissues obtained from 16 patients. The results were further annotated with Kyoto Encyclopedia of Genes and Genomes (KEGG), and Human Metabolome Database, and then were analyzed using modules of pathway analysis and enrichment analysis of MetaboAnalyst 3.0. Based on the analytical techniques, we established the models of principal component analysis (PCA), partial least squares-discriminant analysis (PLS-DA), and orthogonal partial least-squares discriminant analysis (OPLS-DA) which could discriminate PTC from normal thyroid tissue, and found 15 robust differentiated metabolites from two OPLS-DA models. We identified 8 KEGG pathways and 3 pathways of small molecular pathway database which were significantly related to PTC by using pathway analysis and enrichment analysis, respectively, through which we identified metabolisms related to PTC including branched chain amino acid metabolism (leucine and valine), other amino acid metabolism (glycine and taurine), glycolysis (lactate), tricarboxylic acid cycle (citrate), choline metabolism (choline, ethanolamine and glycerolphosphocholine) and lipid metabolism (very-low-density lipoprotein and lowdensity lipoprotein). In conclusion, the PTC was characterized with increased glycolysis and inhibited tricarboxylic acid cycle, increased oncogenic amino acids as well as abnormal choline and lipid metabolism. The findings in this study provide new insights into detailed metabolic changes of PTC, and hold great potential in the treatment of PTC.

\section{Introduction}

Thyroid cancer is the most common cancer in the endocrine system. The incidence of thyroid cancer is increasing every year in the United States, and the increase is not only because of the better diagnosis technique for thyroid cancers. There are estimated to be $\sim 123,000$ people in the world who are diagnosed thyroid cancer cases annually, accounting for approximately over $91.5 \%$ of the cancers of head and neck (1). Notably, papillary thyroid carcinoma (PTC) is the most common type of all thyroid cancers, which accounts for $\sim 80 \%$ of the thyroid cancers. 
Metabolomics is an emerging omics technology, and it can systematically identify and quantify metabolites in a biological sample. The technology development of nuclear magnetic resonance (NMR) spectroscopy and mass spectrometry (MS) allows the simultaneous analysis of various chemicals in the biological sample, and therefore NMR and MS are the favorable platforms for metabolomic analysis (2). Metabolomics has been widely used in studies regarding diseases (3), and it identifies metabolic signatures which are related to the pathogenesis of the diseases (3-5). There are metabolomic studies on PTC based on gas chromatography-mass spectrometry (GC-MS) $(6,7)$. Compared with GC-MS, NMR is generally accepted as the gold standard in metabolite structural elucidation, which is highly selective, non-destructive for chemical detection. Additionally, the metabolite coverage is different between NMR and GC-MS (8). Therefore, different metabolomic information can be provided by NMR and GC-MS. There are limited published metabolomic studies regarding PTC using NMR (9-11), but these studies focused on the diagnosis of PTC, and therefore the detailed metabolic changes especially metabolic pathway changes related to PTC pathogenesis are still largely unknown. Given these facts, it is necessary to adopt NMR based metabolomics technology to study the detailed metabolic changes and disturbed metabolic pathways in PTC.

We used ${ }^{1} \mathrm{H}$ NMR-based metabolomic technique to study the metabolic changes in PTC. We established reliable statistical models which could well discriminate and predict the tumor and normal thyroid tissue. Based on these models and further bioinformatics analysis, we identified detailed metabolic changes and disturbed metabolic pathways in PTC.

\section{Patients and methods}

Basic patient information and sample collection. A total of 16 patients (4 males, 12 females; age range, 19-59 years; tumor size, $1-4.2 \mathrm{~cm}$ ) had undergone surgical thyroidectomy at the First Affiliated Hospital of Nanjing Medical University. Previous metabolomic studies using cancer tissues have proved that similar sample number and characteristics of the population can provide useful information on metabolic changes of cancer (4,9-11). Matched normal thyroid tissues and tumor tissues were obtained from the same PTC patients. The diagnosis was confirmed by histopathologic evaluation based on the established criteria of WHO (12), which was conducted independently by two pathologists. The analyzed tumor tissues which contained over $90 \%$ cancer cells were obtained by using microdissection, and normal tissues were not connected by follicular adenomas (FAs) or PTC. The patients were not received radiation therapy or neo-adjuvant chemotherapy before operation. All tissues were immediately frozen in liquid nitrogen, and then stored at $-80^{\circ} \mathrm{C}$ until NMR analysis. The study was approved by the Ethics Committee of the First Affiliated Hospital of Nanjing Medical University, and each participant signed an informed consent.

${ }^{1} H$ NMR spectroscopy based metabolomic analysis. Metabolomic analysis was conducted according to metabolomic procedure for NMR spectroscopy of tissues (13). The metabolomic analysis was performed with Bruker Avance III 600 NMR spectrometer. Tissue samples were placed in a $4 \mathrm{~mm}$ rotor, and $5 \mu \mathrm{l}$ aliquot of deuterium oxide

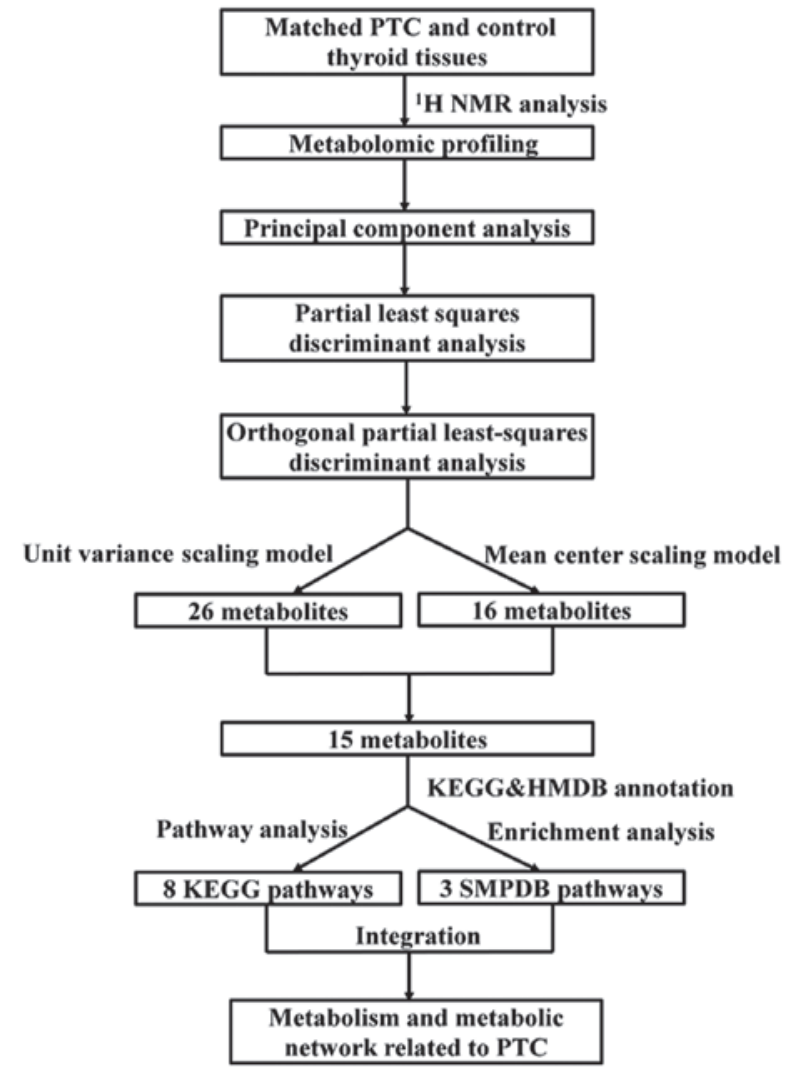

Figure 1. Strategy for the metabolomic study regarding papillary thyroid carcinoma (PTC).

was added into the rotor. The resonance frequency of ${ }^{1} \mathrm{H}$ was $400 \mathrm{MHz}$, and the experimental temperature was $298 \mathrm{~K}$. A Carr-Purcell-Meiboom-Gill (CPMG) filter was included in the pulse sequence to reduce broad resonances related to background or macromolecules. After the fourier transformation, the spectra was manually phased and baseline corrected, and then referenced to lactic acid $\mathrm{CH}_{3}$ resonance at $\delta 1.33$.

Multivariate pattern recognition. In order to discriminate the samples according to tissue types and identify potential biomarkers in relation to PTC, principal component analysis (PCA), partial least squares-discriminant analysis (PLS-DA), and the orthogonal partial least-squares discriminant analysis (OPLS-DA) were applied using the normalized NMR data. We identified changed metabolites in PTC by using OPLS-DA statistical model. SIMCA-P + software (v11.0; Umetrics AB, Umea, Sweden) was used for data analysis. The correlation coefficient of $|r|>0.482$ and the variable importance in projection (VIP) value $>1.00$ were used as cut-off value of the statistical significance based on the discrimination significance at the level of $\mathrm{P}=0.05$.

Biological significance interpretation and informatics analysis. To identify detailed metabolic changes and disturbed metabolic pathways, the differentiated metabolites were first annotated with 'Kyoto Encyclopedia of Genes and Genomes' (KEGG, http://www.genome.jp/kegg/ or http://www.kegg.jp/) which is a knowledge library for systematic analysis of metabolite functions and networks and 'Human Metabolome Database' (HMDB, http://www.hmdb.ca/) which is a complete and compre- 


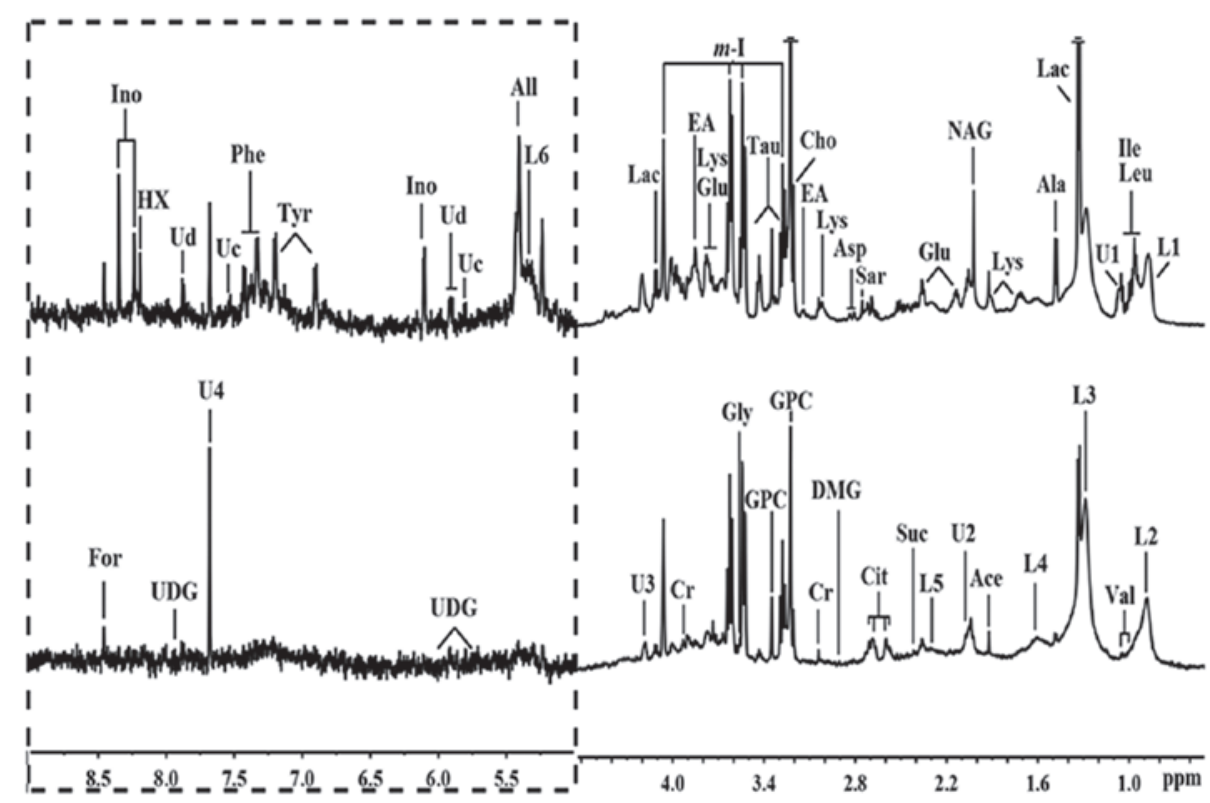

Figure 2. Representative ${ }^{1} \mathrm{H}$ nuclear magnetic resonance (NMR) spectra ( $\delta 0.6-4.6$ and $\left.\delta 5.0-9.0\right)$ of thyroid obtained from papillary thyroid carcinoma (PTC) (above) and normal thyroid tissue (below). The region of $85.0-9.0$ (in the dashed box) was magnified 10 times compared with corresponding region of $\delta 0.6-4.6$ for the purpose of clarity. Ace, acetate; Ala, alanine; All, allantoin; Asp, aspartate; Cho, choline; Cit, citrate; Cr, creatine; EA, ethanolamine; For, formate; Glu, glutamate; Gly, glycine; GPC, glycerolphosphocholine; HX, hypoxanthine; Ile, isoleucine; Ino, inosine; L1: $\mathrm{LDL}_{2} \mathrm{CH}_{3}-\left(\mathrm{CH}_{2}\right)_{\mathrm{n}} ; \mathrm{L}_{2}: \mathrm{VLDL}_{\mathrm{C}} \mathrm{CH}_{3}-(\mathrm{CH})_{\mathrm{n}}-; \mathrm{L} 3$ : VLDL, $\mathrm{CH}_{3}-\left(\mathrm{CH}_{2}\right)_{n}-$; L4: VLDL, - $\mathrm{CH}_{2}-\mathrm{CH}_{2}-\mathrm{C}=\mathrm{O}$; L5: lipid, - $\mathrm{CH}_{2}-\mathrm{C}=\mathrm{O}$; L6: lipid, -CH=CH-; Lac, lactate; Leu, leucine; Lys, lysine; m-I, myo-inositol; DMG, $N, N$-dimethylglycine; NAG, $N$-acetyl glycoprotein signals; Phe, phenylalanine; Sar, sarcosine; Suc, succinate; Tau, taurine; Tyr, tyrosine; U, unknown; Uc, uracil; Ud, uridine; UDG, uridine diphosphate glucose; Val, valine.

hensive database on metabolomics with metabolite biological interpretation $(14,15)$. Further, we conducted informatics analysis by MetaboAnalyst 3.0 (http://www.metaboanalyst. $\mathrm{ca} /$ MetaboAnalyst/) which is built by R software (v3.2.2) (16). MetaboAnalyst combines the results from powerful pathway enrichment analysis with the pathway topology analysis to discover the relevant pathways. Two modules of MetaboAnalyst including pathway analysis and enrichment analysis were used. The module of pathway analysis was based on the KEGG database; the enrichment analysis was conducted based on Small Molecule Pathway Database (SMPDB, http://smpdb.ca/) (17).

\section{Results}

Metabolomic profiling. Fig. 1 shows the workflow of this metabolomic study. The metabolomic profiles obtained from PTC and normal thy roid tissue are shown in Fig. 2. The metabolic profiling covered branched chain amino acids (isoleucine, leucine and valine), other proteinogenic amino acids (alanine, phenylalanine, tyrosine, glycine, aspartate, glutamate and lysine), and product of amino acid (creatine) as well as amino acid derivatives (sarcosine and taurine). Metabolites involved in the nucleotide metabolism (hypoxanthine, uracil, uridine and allantoin), glycolysis (lactate), tricarboxylic acid cycle (citrate and succinate), choline metabolism (choline, ethanolamine, glycerolphosphocholine and $N, N$-dimethylglycine) and lipid metabolism [very-low-density lipoprotein (VLDL), low-density lipoprotein (LDL) and lipids] were also profiled.PCA. PCA is the most commonly used algorithm in metabolomics studies (18). We adopted PCA in the study to process the NMR data based on a mean center-scaling model, which is an unsupervised projection method used to visualize the dataset and display

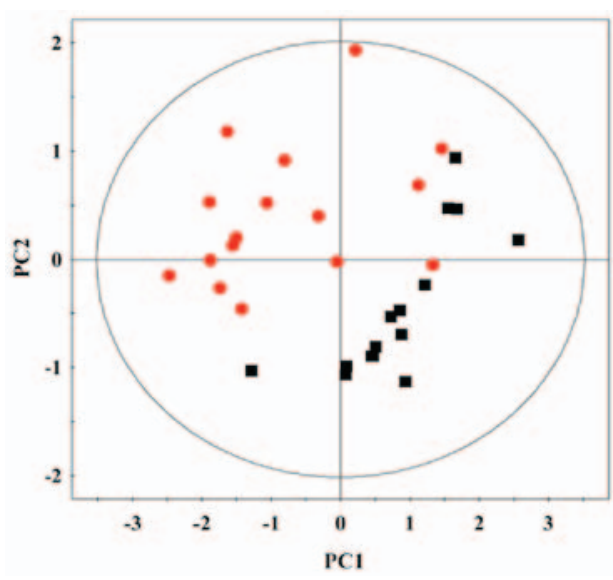

Figure 3. Principal component analysis (PCA) score plot based on ${ }^{1} \mathrm{H}$ nuclear magnetic resonance (NMR) spectra of thyroid obtained from papillary thyroid carcinoma (PTC) (red) and normal tissue (black) $\left(\mathrm{R}^{2} \mathrm{X}=0.79, \mathrm{Q}^{2}=0.75\right)$.

the intrinsic similarity and difference in the dataset. As shown in Fig. 3 (19), the PTC tissues were clearly discriminated from normal tissues using PCA model, indicating dramatic metabolic changes in PTC. The PCA model descriptors $\left(\mathrm{R}^{2} \mathrm{X}, \mathrm{Q}^{2}\right)$ were $0.79 / 0.75$, indicating the model was reliable.

$P L S$-DA. PLS-DA was further conducted to evaluate the data variance between PTC and normal tissue. As shown in Fig. 4A (20), PLS-DA score plot showed a statistically significant discrimination between the two groups based on the mean center-scaling model $\left(\mathrm{R}^{2} \mathrm{X}=0.79, \mathrm{R}^{2} \mathrm{Y}=0.71, \mathrm{Q}^{2}=0.66\right)$. Similar significant discrimination between the two groups was also observed based on the unit variance scaling model $\left(\mathrm{R}^{2} \mathrm{X}=0.26, \mathrm{R}^{2} \mathrm{Y}=0.82, \mathrm{Q}^{2}=0.71\right.$ ) (Fig. 4B) (20). To test the 
A

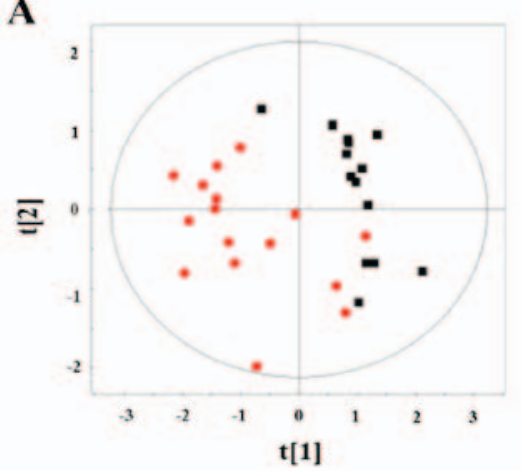

$\mathbf{B}$

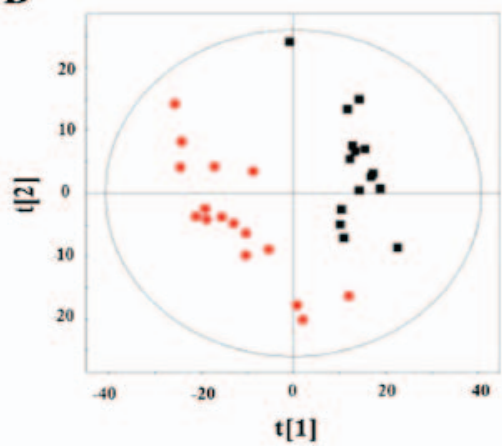

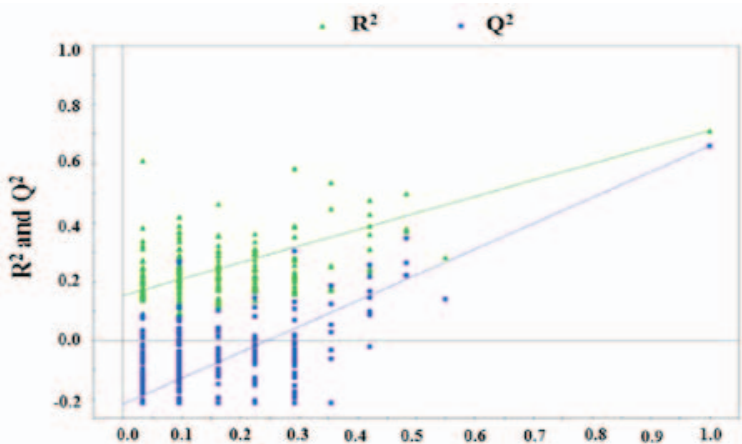

Correlation coefficient between original and permuted data

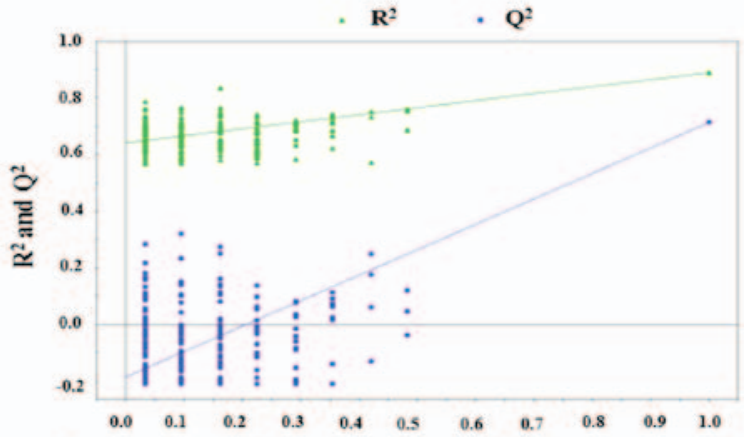

Correlation coefficient between original and permuted data

Figure 4. Partial least squares-discriminant analysis (PLS-DA) score plot based on ${ }^{1} \mathrm{H}$ nuclear magnetic resonance (NMR) spectra of thyroid obtained from papillary thyroid carcinoma (PTC) (red) and normal tissue (black). (A) Mean center-scaling model $\left(\mathrm{R}^{2} \mathrm{X}=0.79, \mathrm{R}^{2} \mathrm{Y}=0.71, \mathrm{Q}^{2}=0.66\right.$ ); (B) Unit variance scaling model $\left(\mathrm{R}^{2} \mathrm{X}=0.26, \mathrm{R}^{2} \mathrm{Y}=0.82, \mathrm{Q}^{2}=0.71\right)$.
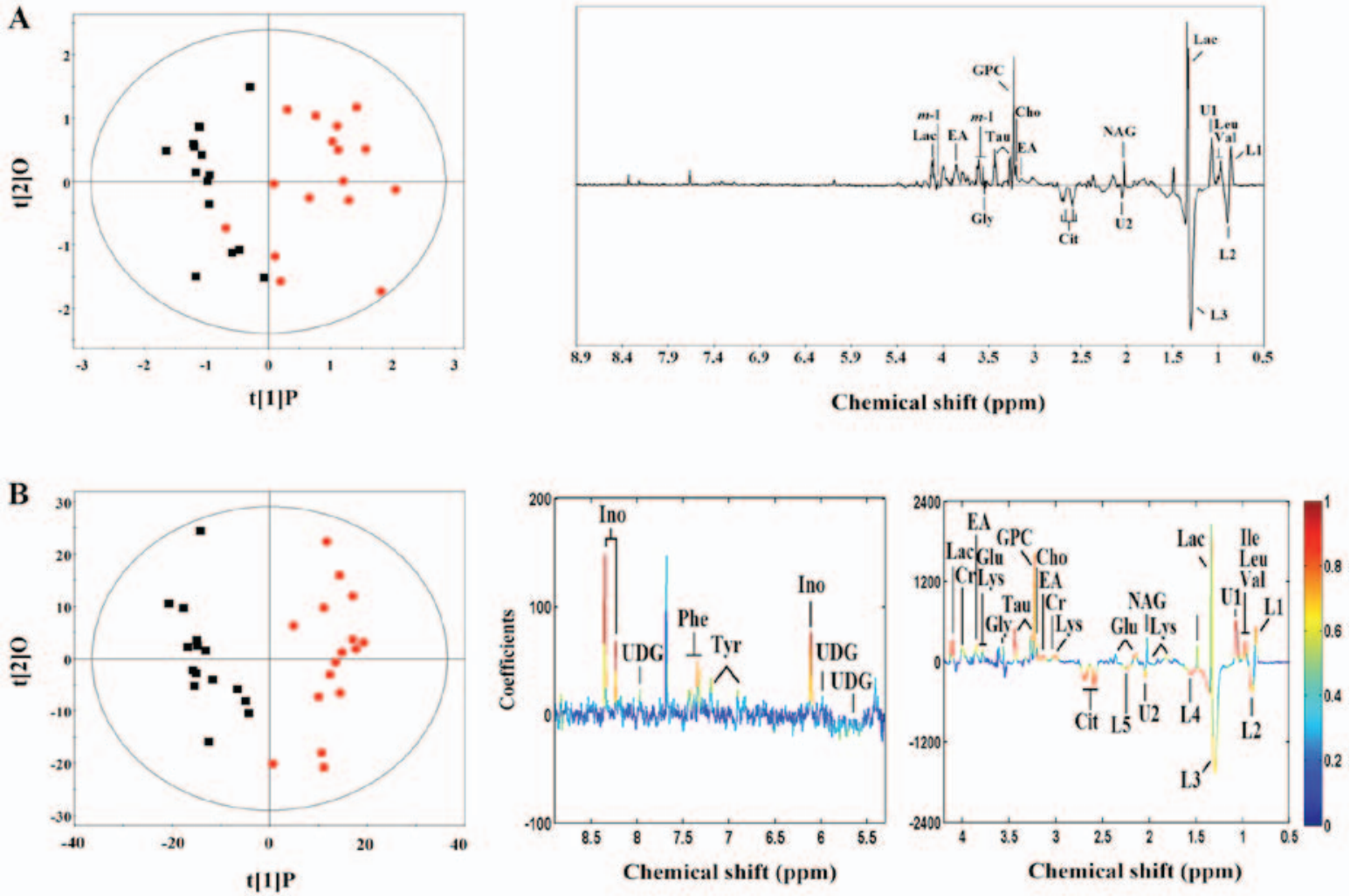

Figure 5. Orthogonal partial least-squares discriminant analysis (OPLS-DA) score plots derived from ${ }^{1} \mathrm{H}$ nuclear magnetic resonance (NMR) spectra (left panel) and corresponding ${ }^{1} \mathrm{H}$ NMR spectra with indicated differential metabolites obtained from different groups (right panel). (A) The mean center-scaling model $\left(\mathrm{R}^{2} \mathrm{X}=0.79, \mathrm{Q}^{2}=0.66\right)$; peaks in the positive direction of the ${ }^{1} \mathrm{H}$ NMR spectra indicated metabolites those were more abundant in the papillary thyroid carcinoma (PTC) groups. (B) The unit variance scaling mode $\left(R^{2} X=0.26, Q^{2}=0.77\right)$; the color map shows the significance of metabolite variations between the two classes. Metabolites were more abundant in the PTC presented as peaks in the positive direction. The region of $85.0-9.0$ was magnified 10 times compared with corresponding region of $80.6-4.6$ for the purpose of clarity. Abbreviations of the assignments are shown in Fig. 2. 
Table I. OPLS-DA coefficients derived from the NMR data of metabolites in thyroid obtained from PTC and normal tissue in the unit variance scaling mode.

\begin{tabular}{|c|c|}
\hline Metabolites & $\mathrm{r}^{\mathrm{a}}$ \\
\hline Alanine: $1.48\left(\mathrm{~d}^{\mathrm{b}}\right)$ & 0.738 \\
\hline Choline: $3.20(\mathrm{~s})$ & 0.758 \\
\hline Citrate: $2.57(\mathrm{~d}), 2.69(\mathrm{~d})$ & -0.792 \\
\hline Creatine: $3.04(\mathrm{~s}), 3.93(\mathrm{~s})$ & 0.594 \\
\hline Ethanolamine: $3.14(\mathrm{t}), 3.85(\mathrm{t})$ & 0.858 \\
\hline Glutamate: $2.15(\mathrm{~m}), 2.35(\mathrm{~m}), 3.78(\mathrm{t})$ & 0.831 \\
\hline Glycine: $3.56(\mathrm{~s})$ & 0.566 \\
\hline Glycerophosphocholine: $3.23(\mathrm{~s}), 3.35(\mathrm{~s})$ & 0.754 \\
\hline Isoleucine: $0.94(\mathrm{~d}), 1.01(\mathrm{~d})$ & 0.597 \\
\hline Inosine: $6.10(\mathrm{~d}), 8.23(\mathrm{~s}), 8.34(\mathrm{~s})$ & 0.897 \\
\hline $\mathrm{L} 1, \mathrm{LDL}, \underline{\mathrm{C}}_{3}-\left(\mathrm{CH}_{2}\right)_{\mathrm{n}}-: 0.86(\mathrm{br})$ & 0.732 \\
\hline $\mathrm{L} 2, \mathrm{VLDL}, \mathrm{CH}_{3}-\left(\mathrm{CH}_{2}\right)_{\mathrm{n}}:: 0.90(\mathrm{br})$ & -0.762 \\
\hline $\mathrm{L} 3, \mathrm{VLDL}, \mathrm{CH}_{3}-\left(\mathrm{CH}_{2}\right)_{\mathrm{n}}-: 1.29(\mathrm{br})$ & -0.628 \\
\hline $\mathrm{L} 4, \mathrm{VLDL},-\mathrm{C}_{2}-\mathrm{CH}_{2}-\mathrm{C}=\mathrm{O}: 1.59(\mathrm{br})$ & -0.822 \\
\hline L5, Lipid, $-\mathrm{CH}_{2}-\mathrm{C}=\mathrm{O}: 2.26(\mathrm{br})$ & -0.738 \\
\hline Lactate: $1.33(\mathrm{~d}), 4.11(\mathrm{q})$ & 0.893 \\
\hline Leucine: $0.96(t)$ & 0.767 \\
\hline Lysine: $1.72(\mathrm{~m}), 1.91(\mathrm{~m}), 3.01(\mathrm{~m}), 1.76(\mathrm{t})$ & 0.750 \\
\hline$N$-acetyl glycoprotein signals: $2.03(\mathrm{~s})$ & 0.520 \\
\hline Phenylalanine: $7.33(\mathrm{~d}), 7.37(\mathrm{t}), 7.43(\mathrm{dd})$ & 0.759 \\
\hline Taurine: $3.28(\mathrm{t}), 3.43(\mathrm{t})$ & 0.861 \\
\hline Tyrosine: $6.90(\mathrm{~d}), 7.20(\mathrm{~d})$ & 0.532 \\
\hline Unknown-1: $1.07(\mathrm{~m})$ & 0.854 \\
\hline Unknown-2: 2.06(m) & -0.679 \\
\hline $\begin{array}{l}\text { Uridine diphosphate glucose: } 5.61(\mathrm{dd}) \text {, } \\
5.98(\mathrm{~m}), 7.96(\mathrm{~d})\end{array}$ & 0.569 \\
\hline Valine: $0.99(\mathrm{~d}), 1.04(\mathrm{~d})$ & 0.889 \\
\hline \multicolumn{2}{|c|}{$\begin{array}{l}\text { a'Correlation coefficients, positive and negative signs indicate positive and } \\
\text { negative correlation in the concentrations, respectively. The correlation } \\
\text { coefficient of }|r|>0.482 \text { was used as the cut-off value for the statistical sig- } \\
\text { nificance based on the discrimination significance at the level of } P=0.05 \\
\text { and df (degree of freedom) }=15 \text {. buultiplicity: } \mathrm{s} \text {, singlet; d, doublet; } \\
\text { t, triplet; q, quartet; dd, doublet of doublets; } \mathrm{m} \text {, multiplet; br, broad reso- } \\
\text { nance; OPLS-DA, orthogonal partial least-squares discriminant analysis; } \\
\text { NMR, nuclear magnetic resonance; PTC, papillary thyroid carcinoma. }\end{array}$} \\
\hline
\end{tabular}

validity of the PLS-DA models, the robustness of the models was assessed using a 200-permutations validation model, which showed that the originally observed separation was not due to a random effect, as the predictive discrimination values of the random models were all lower than that of the original model.

$O P L S$-DA. AdditionalOPLS-DA was conducted to establish the mean center-scaling model and the unit variance scaling model. The mean center-scaling model descriptors $\left(\mathrm{R}^{2} \mathrm{X}, \mathrm{Q}^{2}\right)$ were $0.79 / 0.66$ (Fig. 5A), and the unit variance scaling mode descriptors $\left(\mathrm{R}^{2} \mathrm{X}, \mathrm{Q}^{2}\right)$ were 0.26/0.77 (Fig. 5B), indicating these models were reliable.

Differential metabolites in PTC. Based on the unit variance scaling OPLS-DA model, there were 26 differential
Table II. VIP value derived from the NMR data of metabolites in thyroid obtained from PTC and normal tissue in the mean center-scaling model.

\begin{tabular}{lr}
\hline Metabolites & VIP $^{\mathrm{a}}$ \\
\hline Lactate: $1.33\left(\mathrm{~d}^{\mathrm{b}}\right), 4.11(\mathrm{q})$ & 10.81 \\
L3, VLDL, $\mathrm{CH}_{3}-\left(\mathrm{CH}_{2}\right)_{\mathrm{n}}-: 1.29(\mathrm{~d})$ & 9.81 \\
Glycerophosphocholine: $3.23(\mathrm{~s}), 3.35(\mathrm{~s})$ & 9.24 \\
Unknown-1: $1.07(\mathrm{~m})$ & 3.63 \\
L1, LDL, $\mathrm{CH}_{3}-\left(\mathrm{CH}_{2}\right)_{\mathrm{n}}-:$ : $0.86(\mathrm{br})$ & 3.05 \\
Valine: $0.99(\mathrm{~d}), 1.04(\mathrm{~d})$ & 2.81 \\
L2, VLDL, $\mathrm{CH}_{3}-\left(\mathrm{CH}_{2}\right)_{\mathrm{n}}-:$ 0.90(br) & 2.62 \\
Taurine: $3.28(\mathrm{t}), 3.43(\mathrm{t})$ & 2.44 \\
$N$-acetyl glycoprotein signals: $2.03(\mathrm{~s})$ & 2.29 \\
Myo-inositol: $3.56(\mathrm{dd}), 3.63(\mathrm{dd}), 4.06(\mathrm{t})$ & 2.19 \\
Citrate: $2.57(\mathrm{~d}), 2.69(\mathrm{~d})$ & 1.81 \\
Leucine: $0.96(\mathrm{t})$ & 1.63 \\
Ethanolamine: $3.14(\mathrm{t}), 3.85(\mathrm{t})$ & 1.63 \\
Choline: $3.20(\mathrm{~s})$ & 1.26 \\
Unknown-2: $2.06(\mathrm{~m})$ & 1.13 \\
Glycine: $3.56(\mathrm{~s})$ & 1.04 \\
\hline
\end{tabular}

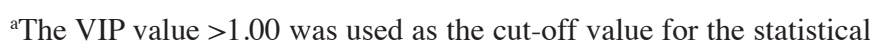
significance based on the discrimination significance at the level of $\mathrm{P}=0.05$. 'Multiplicity: s, singlet; $\mathrm{d}$, doublet; $\mathrm{t}$, triplet; $\mathrm{q}$, quartet; $\mathrm{dd}$, doublet of doublets; $\mathrm{m}$, multiplet; br, broad resonance; VIP, variable importance in projection; NMR, nuclear magnetic resonance; PTC, papillary thyroid carcinoma.

metabolites identified. The differential metabolites in PTC are presented in Table I and Fig. 5B. To find the robustly changed metabolites, we verified the changed metabolites in the mean center-scaling model, which identified 16 differential metabolites (Table II and Fig. 5A), among which 15 consistently changed metabolites were found (Table III).

Metabolic pathway analysis and biological significance interpretation. The 15 metabolites related to PTC with robust results were then annotated with KEGG and HMDB (Table III). The results were submitted to MetaboAnalyst to show the statistical analysis results of informatics analysis. The result of pathway analysis is shown in Table IV and Fig. 6A, which found 8 pathways including glycerophospholipid metabolism, aminoacyl-tRNA biosynthesis, valine, leucine and isoleucine biosynthesis, propanoate metabolism, nitrogen metabolism, valine, leucine and isoleucine degradation, primary bile acid biosynthesis, and glycine, serine and threonine metabolism were significantly related to PTC. Furthermore, in order to expand the understanding of metabolic pathway related to PTC, the module of enrichment analysis of MetaboAnalyst was used, which found 3 additional pathways including protein biosynthesis, phospholipid biosynthesis and methionine metabolism significantly related to PTC (Table V and Fig. 6B). Finally, based on biological significance and the above informatics analysis, the metabolic network related to PTC was built (Fig. 7), which indicated the key metabolisms related to PTC including branched chain amino acid metabolism 
Table III. The consistently changed metabolites in thyroid obtained from PTC and normal tissue in the OPLS-DA models.

\begin{tabular}{|c|c|c|c|c|c|}
\hline Metabolites & KEGG & HMDB & $\mathrm{r}^{\mathrm{a}}$ & VIP & Pathway \\
\hline Choline & $\mathrm{C} 00114$ & HMDB0097 & 0.758 & 1.26 & Choline metabolism \\
\hline Ethanolamine & C00189 & HMDB0149 & 0.858 & 1.63 & Choline metabolism \\
\hline Glycerophosphocholine & $\mathrm{C} 00670$ & HMDB0086 & 0.754 & 9.24 & Choline metabolism \\
\hline Lactate & $\mathrm{C} 00186$ & HMDB0190 & 0.893 & 10.81 & Glycolysis \\
\hline Citrate & $\mathrm{C} 00158$ & HMDB0094 & -0.792 & 1.81 & Tricarboxylic acid cycle \\
\hline Leucine & $\mathrm{C} 00123$ & HMDB0687 & 0.767 & 1.63 & Branched chain amino acid metabolism \\
\hline Valine & $\mathrm{C} 00183$ & HMDB0883 & 0.889 & 2.81 & Branched chain amino acid metabolism \\
\hline Glycine & $\mathrm{C} 00037$ & HMDB0123 & 0.566 & 1.04 & Other amino acid metabolism \\
\hline Taurine & $\mathrm{C} 00245$ & HMDB0251 & 0.861 & 2.44 & Other amino acid metabolism \\
\hline L1, LDL & NA & NA & 0.732 & 3.05 & Lipid metabolism \\
\hline L2, VLDL & NA & NA & -0.762 & 2.62 & Lipid metabolism \\
\hline L3, VLDL & NA & NA & -0.628 & 9.81 & Lipid metabolism \\
\hline $\begin{array}{l}N \text {-acetyl glycoprotein } \\
\text { signals }\end{array}$ & NA & NA & 0.520 & 2.29 & NA \\
\hline Unknown-1: 1.07(m) & NA & NA & 0.854 & 3.63 & NA \\
\hline Unknown-2: 2.06(m) & NA & NA & -0.679 & 1.13 & NA \\
\hline
\end{tabular}

${ }^{a}$ Correlation coefficients derived from the unit variance scaling model, positive and negative signs indicate positive and negative correlation in the concentrations, respectively. The correlation coefficient of $|r|>0.482$ was used as the cut-off value for the statistical significance based on the discrimination significance at the level of $\mathrm{P}=0.05$ and $\mathrm{df}=15$. ${ }^{\mathrm{b}}$ The VIP value derived from the mean center-scaling model $>1.00$ was used as the cut-off value for the statistical significance based on the discrimination significance at the level of $\mathrm{P}=0.05$. PTC, papillary thyroid carcinoma; OPLS-DA, orthogonal partial least-squares discriminant analysis; NA, not available; df, degrees of freedom.

Table IV. Pathway analysis of metabolic changes in PTC. ${ }^{a}$

\begin{tabular}{|c|c|c|c|c|c|}
\hline KEGG pathway & Total & Expected & Hits & Impact & P-value \\
\hline Glycerophospholipid metabolism & 39 & 0.146 & 3 & 0.087 & 3.09E-04 \\
\hline Aminoacyl-tRNA biosynthesis & 75 & 0.280 & 3 & 0.000 & $2.13 \mathrm{E}-03$ \\
\hline Valine, leucine and isoleucine biosynthesis & 27 & 0.101 & 2 & 0.027 & 4.16E-03 \\
\hline Propanoate metabolism & 35 & 0.131 & 2 & 0.000 & $6.94 \mathrm{E}-03$ \\
\hline Nitrogen metabolism & 39 & 0.146 & 2 & 0.000 & 8.57E-03 \\
\hline Valine, leucine and isoleucine degradation & 40 & 0.150 & 2 & 0.022 & $9.01 \mathrm{E}-03$ \\
\hline Primary bile acid biosynthesis & 47 & 0.176 & 2 & 0.016 & $1.23 \mathrm{E}-02$ \\
\hline Glycine, serine and threonine metabolism & 48 & 0.179 & 2 & 0.188 & $1.28 \mathrm{E}-02$ \\
\hline Cyanoamino acid metabolism & 16 & 0.060 & 1 & 0.000 & $5.84 \mathrm{E}-02$ \\
\hline Citrate cycle (TCA cycle) & 20 & 0.075 & 1 & 0.063 & 7.25E-02 \\
\hline Taurine and hypotaurine metabolism & 20 & 0.075 & 1 & 0.331 & $7.25 \mathrm{E}-02$ \\
\hline Ether lipid metabolism & 23 & 0.086 & 1 & 0.000 & 8.29E-02 \\
\hline Thiamine metabolism & 24 & 0.090 & 1 & 0.000 & 8.64E-02 \\
\hline Pantothenate and CoA biosynthesis & 27 & 0.101 & 1 & 0.000 & $9.67 \mathrm{E}-02$ \\
\hline Glycolysis or gluconeogenesis & 31 & 0.116 & 1 & 0.000 & $1.10 \mathrm{E}-01$ \\
\hline Pyruvate metabolism & 32 & 0.120 & 1 & 0.138 & $1.14 \mathrm{E}-01$ \\
\hline Methane metabolism & 34 & 0.127 & 1 & 0.000 & $1.20 \mathrm{E}-01$ \\
\hline Glutathione metabolism & 38 & 0.142 & 1 & 0.000 & $1.34 \mathrm{E}-01$ \\
\hline Lysine degradation & 47 & 0.176 & 1 & 0.000 & $1.63 \mathrm{E}-01$ \\
\hline Glyoxylate and dicarboxylate metabolism & 50 & 0.187 & 1 & 0.003 & $1.72 \mathrm{E}-01$ \\
\hline Purine metabolism & 92 & 0.344 & 1 & 0.000 & $2.96 \mathrm{E}-01$ \\
\hline Porphyrin and chlorophyll metabolism & 104 & 0.389 & 1 & 0.000 & 3.28E-01 \\
\hline
\end{tabular}

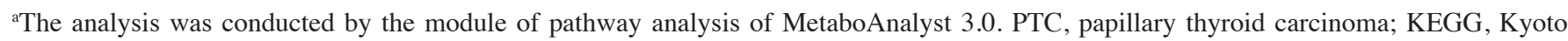
Encyclopedia of Genes and Genomes. 
A

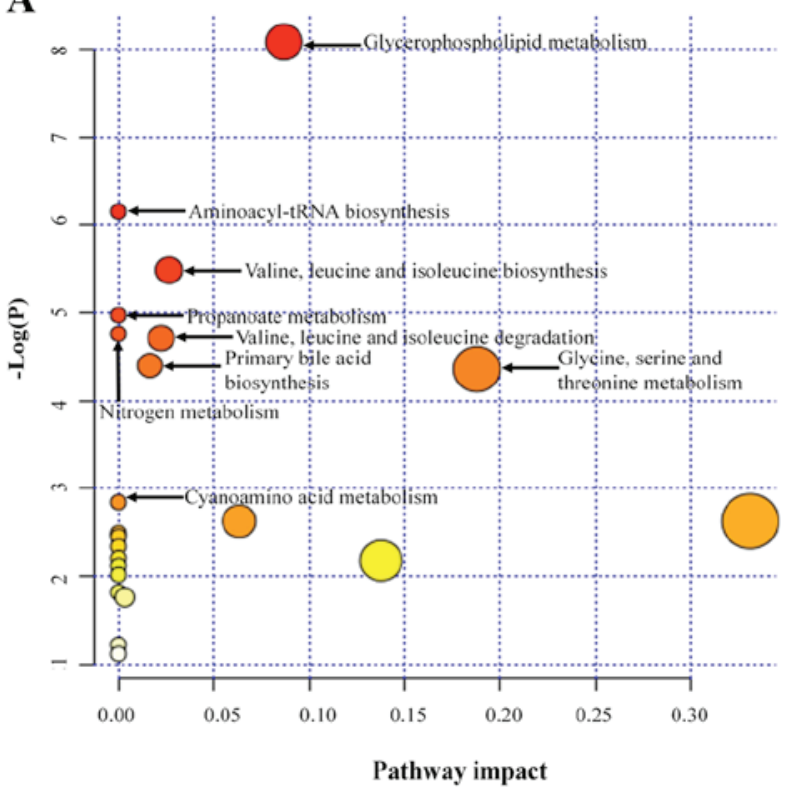

B

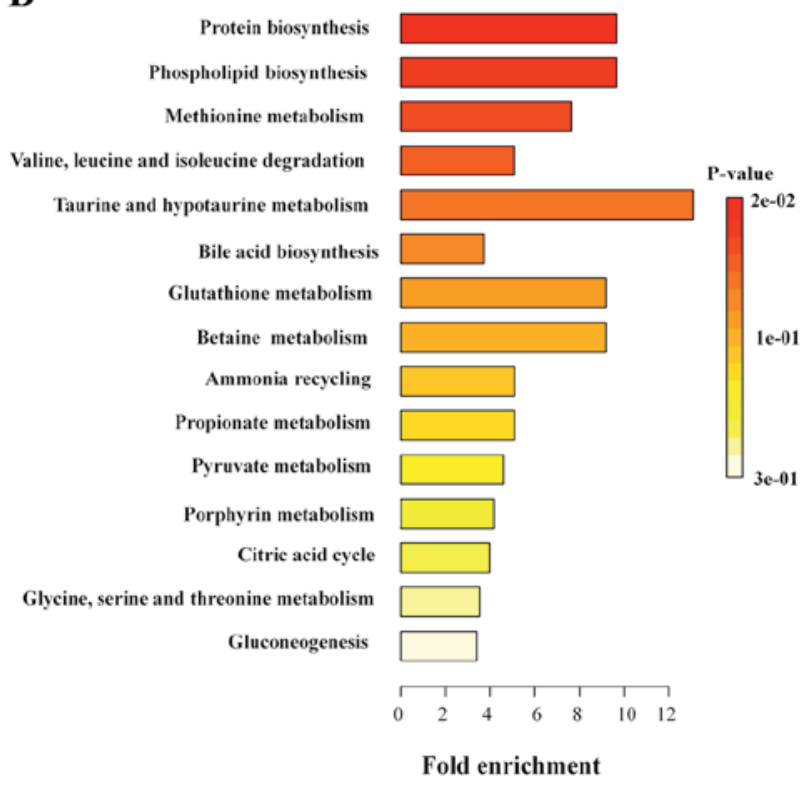

Figure 6. The results of pathway analysis and enrichment analysis of the metabolomic data. (A) Pathway analysis based on Kyoto Encyclopedia of Genes and Genomes (KEGG). (B) Enrichment analysis based on Small Molecule Pathway Database (SMPDB).

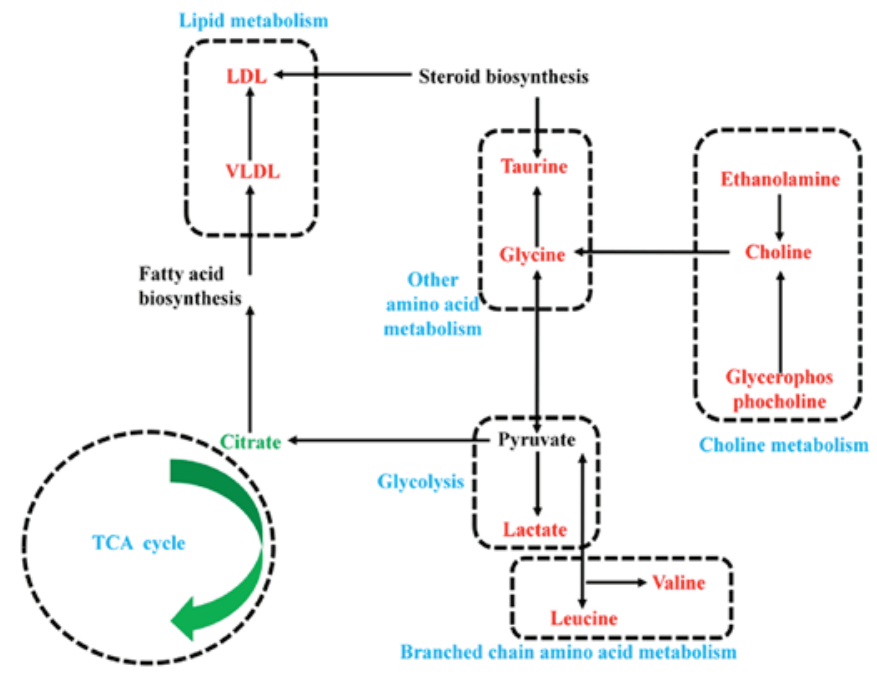

Figure 7. The metabolic network in papillary thyroid carcinoma (PTC). Red shows an increase in PTC; green, shows a decrease in PTC; black, shows no available data; blue, shows relevant metabolic pathway in PTC. TCA, tricarboxylic acid.

(leucine and valine), other amino acid metabolism (glycine and taurine), glycolysis (lactate), tricarboxylic acid cycle (citrate), choline metabolism (choline, ethanolamine and glycerolphosphocholine) and lipid metabolism (VLDL and LDL).

\section{Discussion}

Based on the rapid development of analytical techniques, metabolomics has been applied in many fields such as biochemical and clinical study (21-24). In the study of human diseases, metabolomics takes advantage of novel biomarker exploration and pathophysiological interpretation at the molecular level (21-24). However, until now, there are only NMR-based metabolomic studies focusing on the diagnosis of PTC (9-11), and therefore
Table V. Pathway enrichment of metabolic changes in PTC. ${ }^{a}$

Pathway from SMPDB Total Expected Hits P-value

\begin{tabular}{lllll}
\hline Protein biosynthesis & 19 & 0.207 & 2 & $1.64 \mathrm{E}-02$ \\
Phospholipid biosynthesis & 19 & 0.207 & 2 & $1.64 \mathrm{E}-02$ \\
Methionine metabolism & 24 & 0.262 & 2 & $2.58 \mathrm{E}-02$ \\
Valine, leucine and & 36 & 0.393 & 2 & $5.50 \mathrm{E}-02$
\end{tabular}

isoleucine degradation

$\begin{array}{lllll}\text { Taurine and hypotaurine } & 7 & 0.076 & 1 & 7.42 \mathrm{E}-02\end{array}$ metabolism

$\begin{array}{lllll}\text { Bile acid biosynthesis } & 49 & 0.535 & 2 & 9.52 \mathrm{E}-02\end{array}$

$\begin{array}{lllll}\text { Glutathione metabolism } & 10 & 0.109 & 1 & 1.04 \mathrm{E}-01\end{array}$

$\begin{array}{lllll}\text { Betaine metabolism } & 10 & 0.109 & 1 & 1.04 \mathrm{E}-01\end{array}$

$\begin{array}{lllll}\text { Ammonia recycling } & 18 & 0.196 & 1 & 1.81 \mathrm{E}-01\end{array}$

$\begin{array}{lllll}\text { Propanoate metabolism } & 18 & 0.196 & 1 & 1.81 \mathrm{E}-01\end{array}$

$\begin{array}{lllll}\text { Pyruvate metabolism } & 20 & 0.218 & 1 & 1.99 \mathrm{E}-01\end{array}$

$\begin{array}{lllll}\text { Porphyrin metabolism } & 22 & 0.240 & 1 & 2.17 \mathrm{E}-01\end{array}$

$\begin{array}{lllll}\text { Citric acid cycle } & 23 & 0.251 & 1 & 2.26 \mathrm{E}-01\end{array}$

$\begin{array}{lllll}\text { Glycine, serine and } & 26 & 0.284 & 1 & 2.51 \mathrm{E}-01\end{array}$

threonine metabolism

$\begin{array}{lllll}\text { Gluconeogenesis } & 27 & 0.295 & 1 & 2.60 \mathrm{E}-01\end{array}$

${ }^{a}$ The analysis was conducted by the module of enrichment analysis of MetaboAnalyst 3.0. PTC, papillary thyroid carcinoma; SMPDB, Small Molecule Pathway Database.

the detailed metabolic changes potentially related to PTC pathogenesis are still largely unknown. In this study, based on the results of PCA, PLS-DA, OPLS-DA models (Figs. 3-5), we first identified key metabolites related to PTC (Table III). Then, after KEGG and HMDB annotation and following pathway and enrichment analysis, we found significant metabolic pathways 
related to PTC (Fig. 6), through which we found the metabolic network related to PTC, indicating the metabolic changes potentially related to PTC pathogenesis (Fig. 7).

Robust changes of metabolite levels in PTC included increased lactate, which participates in significantly changed KEGG pathway of propanoate metabolism directly related to glycolysis. The significant increase of lactate in other cancers such as colon cancer and prostate cancer has been reported in previous studies $(25,26)$. This metabolic response in PTC indicated an increase of the glycolytic flux due to hypoxia and ischemia in the tumor tissues or the consequence of the so-called 'Warburg effect', producing more waste products such as lactate (27). Previous GC-MS based metabolomics study did not monitor lactate but found the increased expression of the gene LDHA encoding lactate dehydrogenase which catalyzes the synthesis of lactate in PTC (7), which was consistent with the present study. Citrate which was a significantly decreased metabolite in PTC tissue participates in the tricarboxylic acid cycle which is directly related to glycolysis. Previous study also revealed that the decreased tricarboxylic acid cycle was accompanied by increased glycolytic flux in gastric cardia cancer due to decreased pyruvic acid efflux into the tricarboxylic acid cycle (28). Normal tricarboxylic acid cycle may have an inhibitory effect on cancer progression (28). Collectively, these findings revealed increased glycolysis and inhibited tricarboxylic acid cycle in PTC, which may be biologically related to PTC pathogenesis.

Notably, the robust changes of amino acids in PTC include leucine and valine. Leucine and valine were also included in significantly changed KEGG pathway such as aminoacyl-tRNA biosynthesis, and valine, leucine and isoleucine biosynthesis and degradation as well as SMPDB pathway including protein biosynthesis. Branched-chain amino acids include isoleucine, leucine and valine. Recent metabolomics studies have consistently revealed that branched-chain amino acids is positively related to obesity (29) and diabetes $(23,29)$, which are both endocrine abnormalities and associated with the risk of PTC (30). In the unit variance scaling mode, isoleucine was also identified as the biomarker with increased level in PTC. Additionally, isoleucine and leucine have been identified as tumor promoters of bladder cancer (31). Glycine is involved in the body's production of DNA, phospholipids and collagen, as well as in release of energy. It participates in significantly changed KEGG pathways such as glycine, serine and threonine metabolism. It is reported that glycine plays an important role in rapid cancer cell proliferation (32). Amino acid derivative, the increase of taurine was found in PTC. Taurine was identified as a possible fingerprint biomarker in nonmuscle invasive bladder cancer (33). It participates in significantly changed KEGG pathways of aminoacyl-tRNA biosynthesis, indicating the abnormal protein biosynthesis in PTC. As suggested by Tessem et al in colon cancer, the increase of taurine may reflect an imbalance in osmolyte function in cancer cells (25). Therefore, the increased levels of branched chain amino acids, glycine and taurine in PTC should attract attention as they may be the oncogenic biomarkers of PTC 29).

Significant KEGG pathway including glycerophospholipid metabolism and SMPDB pathways including phospholipid biosynthesis as well as methionine metabolism both are related to choline metabolism covering choline, ethanolamine and glycerophosphocholine. Glycerophosphorylcholine is a choline derivative and one of the major forms of choline storage. Indeed, the abnormal choline metabolism has been reported in breast cancer (34). As indicated in SMPDB, choline metabolism is related to the body methylation status which is associated with thyroid carcinoma pathogenesis (35). In this study, the metabolites in lipid metabolism including VLDL and LDL also changed in PTC. This finding is consistent with blood lipid profile alterations in another malignant disease, acute lymphoblastic leukemia (36).

It is urgent to seek new biomarkers for thyroid carcinoma. Metabolomic profiles from tissue have the potential to be used in conjunction with current diagnostics to help guide the clinical management of patients with PTC (9-11). Our study not only established robust multivariate analysis models that could discriminate PTC, but also identified robust metabolic signatures of PTC based on different statistical models. The robustly changed metabolites identified in this study may be used as potential biomarkers for PTC, among which consistent results were found on lactate and taurine in another metabolomics study regarding PTC (10).

In conclusion, in this study, we found that the metabolomic profiling could discriminate PTC in conjunction with multivariate analysis, and identified robust metabolic signatures of PTC. After informatics analysis, we found the PTC is characterized with increased glycolysis and inhibited tricarboxylic acid cycle, increased oncogenic amino acids as well as abnormal choline and lipid metabolism, which needs further research to deeply study the underlying mechanism and the usage of the study findings in the intervention of PTC. The findings in this study provide new insights into metabolic changes of PTC, and hold great potential in the treatment of PTC.

\section{Acknowledgements}

Not applicable.

\section{Funding}

The present study was supported by the National Natural Science Foundation (no. 81770773), the Natural Science Foundation of Jiangsu Province (no. BK20171499); the Jiangsu Province Key Medical Talents (co-construction) Program, the Talent Project of '333' Project in Jiangsu Province, the Jiangsu Province Official Hospital Scientific Research Initial Funding (no. RPF201501), the Jiangsu Province Official Hospital Talents Construction Fund Research Project (no. IR2015101), the National Natural Science Foundation (no. 81402713), the Young Scholars of Jiangsu Province (no. BK20140909), the Innovation Fund Project of the State Key Laboratory of Reproductive Medicine (general) (no. SKLRM-GA201802). The funding bodies had no role in the design of the study or collection, analysis, and interpretation of data or in writing the manuscript.

\section{Availability of data and material}

The datasets used and/or analyzed during the current study are available from the corresponding author on reasonable request.

\section{Authors' contributions}

YL performed the metabolomic analysis and drafted the manuscript. MC analyzed and interpreted the metabolomic data, and made substantial contributions to manuscript revision. CL participated in 
sample collection and sample preparation for metabolomic analysis. YX provided analytical tools, and contributed to study design and metabolic network establishment. BX was a major contributor in sample preparation, and participated in the instrumental analysis. YH performed metabolic pathway analysis, and prepared figures. TC participated in multivariate pattern recognition analysis and table preparation. MS collected the sample, and was a major contributor in study design. WT made substantial contributions to conception and design, and was involved in acquisition of data and manuscript revision. All authors read and approved the final manuscript.

\section{Ethics approval and consent to participate}

The study was approved by the Ethics Committee of the First Affiliated Hospital of Nanjing Medical University, and each participant signed an informed consent.

\section{Consent for publication}

The participants provided written informed consent for the publication of any associated data and accompanying images.

\section{Competing interests}

The authors declare that they have no competing interests.

\section{References}

1. Jemal A, Siegel R, Ward E, Hao Y, Xu J, Murray T and Thun MJ: Cancer statistics, 2008. CA Cancer J Clin 58: 71-96, 2008.

2. Cheng LL, Burns MA, Taylor JL, He W, Halpern EF, McDougal WS and Wu CL: Metabolic characterization of human prostate cancer with tissue magnetic resonance spectroscopy. Cancer Res 65: 3030-3034, 2005.

3. Brindle JT, Antti H, Holmes E, Tranter G, Nicholson JK, Bethell HW, Clarke S, Schofield PM, McKilligin E, Mosedale DE et al: Rapid and noninvasive diagnosis of the presence and severity of coronary heart disease using ${ }^{1} \mathrm{H}-\mathrm{NMR}$-based metabonomics. Nat Med 8: 1439-1444, 2002.

4. Sreekumar A, Poisson LM, Rajendiran TM, Khan AP, Cao Q, Yu J, Laxman B, Mehra R, Lonigro RJ, Li Y, et al: Metabolomic profiles delineate potential role for sarcosine in prostate cancer progression. Nature 457: 910-914, 2009.

5. El-Bacha T Struchiner CJ Cordeiro MT, Almeida FC, Marques ET Jr and Da Poian AT: ${ }^{1} \mathrm{H}$ nuclear magnetic resonance metabolomics of plasma unveils liver dysfunction in dengue patients. J Virol 90: 7429-7443, 2016.

6. Shang X, Zhong X and Tian X: Metabolomics of papillary thyroid carcinoma tissues: potential biomarkers for diagnosis and promising targets for therapy. Tumour Biol 37: 11163-11175, 2016

7. Chen M, Shen M, Li Y, Liu C, Zhou K, Hu W, Xu B, Xia Y and Tang W: GC-MS-based metabolomic analysis of human papillary thyroid carcinoma tissue. Int J Mol Med 36: 1607-1614, 2015.

8. Pan $Z$ and Raftery D: Comparing and combining NMR spectroscopy and mass spectrometry in metabolomics. Anal Bioana Chem 387: 525-527, 2007.

9. Jordan KW, Adkins CB, Cheng LL and Faquin WC: Application of magnetic-resonance-spectroscopy-based metabolomics to the fine-needle aspiration diagnosis of papillary thyroid carcinoma. Acta Cytol 55: 584-589, 2011.

10. Miccoli P, Torregrossa L, Shintu L, Magalhaes A, Chandran J, Tintaru A, Ugolini C, Minuto MN, Miccoli M, Basolo F, et al: Metabolomics approach to thyroid nodules: a high-resolution magic-angle spinning nuclear magnetic resonance-based study. Surgery 152: 1118-1124, 2012.

11. Deja S, Dawiskiba T, Balcerzak W, Orczyk-Pawiłowicz M, Głod M, Pawełka D and Młynarz P: Follicular adenomas exhibit a unique metabolic profile. ${ }^{1} \mathrm{H}$ NMR studies of thyroid lesions. PLoS One 8: e84637, 2013.

12. DeLellis RA, Lloyd RV, Heitz PU and Eng C (eds): Organization Classification of Tumours. Pathology and Genetics of Tumours of Endocrine Organs. Vol 8. 3rd edition. IARC Press, Lyon, 2004.

13. Jordan KW, Nordenstam J, Lauwers GY, Rothenberger DA, Alavi K, Garwood M and Cheng LL: Metabolomic characterization of human rectal adenocarcinoma with intact tissue magnetic resonance spectroscopy. Dis Colon Rectum 52: 520-525, 2009.
14. Du J, Yuan Z, Ma Z, Song J, Xie X and Chen Y: KEGG-PATH: Kyoto Encyclopedia of Genes and Genomes-based pathway analysis using a path analysis model. Mol Biosyst 10: 2441-2447, 2014.

15. Wishart DS, Jewison T, Guo AC, Wilson M, Knox C, Liu Y, Djoumbou Y, Mandal R, Aziat F, Dong E, et al: HMDB 3.0 - the Human Metabolome Database in 2013. Nucleic Acids Res 41: D801-D807, 2013.

16. Xia J, Sinelnikov IV, Han B and Wishart DS: MetaboAnalyst 3.0 - making metabolomics more meaningful. Nucleic Acids Res 43: W251-W257, 2015.

17. Frolkis A, Knox C, Lim E, Jewison T, Law V, Hau DD Liu P, Gautam B, Ly S, Guo AC, et al: SMPDB: The Small Molecule Pathway Database. Nucleic Acids Res 38 (Suppl 1): D480-D487, 2010.

18. Anthony ML, Rose VS, Nicholson JK and Lindon JC: Classification of toxin-induced changes in ${ }^{1} \mathrm{H}$ NMR spectra of urine using an artificial neural network. J Pharm Biomed Anal 13: 205-211, 1995.

19. Lamour SD, Straschil U, Saric J and Delves MJ: Changes in metabolic phenotypes of Plasmodium falciparum in vitro cultures during gametocyte development. Malar J 13: 468-478, 2014.

20. Bahety P, Tan YM, Hong Y, Zhang L, Chan EC and Ee PL: Metabotyping of docosahexaenoic acid - treated Alzheimer's disease cell model. PLoS One 9: e90123, 2014

21. Wang Z, Klipfell E, Bennett BJ, Koeth R, Levison BS, Dugar B, Feldstein AE, Britt EB, Fu X, Chung YM, et al: Gut flora metabolism of phosphatidylcholine promotes cardiovascular disease. Nature 472: 57-63, 2011

22. Zhang Y, Dai Y, Wen J, Zhang W, Grenz A, Sun H, Tao L, Lu G, Alexander DC, Milburn MV, et al: Detrimental effects of adenosine signaling in sickle cell disease. Nat Med 17: 79-86, 2011.

23. Wang TJ, Larson MG, Vasan RS, Cheng S, Rhee EP, McCabe E, Lewis GD, Fox CS, Jacques PF, Fernandez C, et al: Metabolite profiles and the risk of developing diabetes. Nat Med 17: 448-453, 2011

24. Chen M,Zhou K, Chen X, Qiao S, Hu Y, Xu B, Xu B, Han X, Tang R, Mao Z, et al: Metabolomic analysis reveals metabolic changes caused by bisphenol A in rats. Toxicol Sci 138: 256-267, 2014.

25. Tessem MB, Selnaes KM, Sjursen W, Tranø G, Giskeødegård GF Bathen TF, Gribbestad IS and Hofsli E: Discrimination of patients with microsatellite instability colon cancer using ${ }^{1} \mathrm{H} H \mathrm{HR}$ MAS MR spectroscopy and chemometric analysis. J Proteome Res 9: 3664-3670, 2010.

26. Tessem MB, Swanson MG, Keshari KR, Albers MJ, Joun D, Tabatabai ZL, Simko JP, Shinohara K, Nelson SJ, Vigneron DB, et al: Evaluation of lactate and alanine as metabolic biomarkers of prostate cancer using ${ }^{1} \mathrm{H}$ HR-MAS spectroscopy of biopsy tissues. Magn Reson Med 60: 510-516, 2008.

27. Koppenol WH, Bounds PL and Dang CV: Otto Warburg's contributions to current concepts of cancer metabolism. Nat Rev Cancer 11: 325-337, 2011.

28. Cai Z, Zhao JS, Li JJ, Peng DN, Wang XY, Chen TL, Qiu YP, Chen PP, Li WJ, Xu LY, et al: A combined proteomics and metabolomics profiling of gastric cardia cancer reveals characteristic dysregulations in glucose metabolism. Mol Cell Proteomics 9: 2617-2628, 2010

29. Newgard CB, An J, Bain JR, Muehlbauer MJ, Stevens RD, Lien LF, Haqq AM, Shah SH, Arlotto M, Slentz CA, et al: A' branched-chain amino acid-related metabolic signature that differentiates obese and lean humans and contributes to insulin resistance. Cell Metab 9: 311-326, 2009.

30. Cheng SP, Chi CW, Tzen CY, Yang TL, Lee JJ, Liu TP and Liu CL: Clinicopathologic significance of leptin and leptin receptor expressions in papillary thyroid carcinoma. Surgery 147: 847-853,2010.

31. Nishio Y, Kakizoe T, Ohtani M, Sato S, Sugimura T and Fukushima S: L-isoleucine and L-leucine: tumor promoters of bladder cancer in rats. Science 231: 843-845, 1986.

32. Jain M, Nilsson R, Sharma S, Madhusudhan N, Kitami T, Souza AL, Kafri R, Kirschner MW, Clish CB and Mootha VK: Metabolite profiling identifies a key role for glycine in rapid cancer cell proliferation. Science 336: 1040-1044, 2012.

33. Srivastava S, Roy R, Singh S, Kumar P, Dalela D, Sankhwar SN, Goel A and Sonkar AA: Taurine - a possible fingerprint biomarker in non-muscle invasive bladder cancer: a pilot study by ${ }^{1} \mathrm{H}$ NMR spectroscopy. Cancer Biomark 6: 11-20, 2010.

34. Katz-Brull R, Seger D, Rivenson-Segal D, Rushkin E and Degani H: Metabolic markers of breast cancer: enhanced choline metabolism and reduced choline-ether-phospholipid synthesis. Cancer Res 62: 1966-1970, 2002.

35. Venkataraman GM, Yatin M, Marcinek R and Ain KB: Restoration of iodide uptake in dedifferentiated thyroid carcinoma: relationship to human $\mathrm{Na}^{+} / \mathrm{I}^{-}$symporter gene methylation status. J Clin Endocrinol Metab 84: 2449-2457, 1999.

36. Halton JM, Nazir DJ, McQueen MJ and Barr RD: Blood lipid profiles in children with acute lymphoblastic leukemia. Cancer 83: 379-384, 1998. 